EPiC Series in Engineering
Volume 3, 2018, Pages 1547-1556
HIC 2018. 13th International
Conference on Hydroinformatics

\title{
Development of Decision Support Tool for Evaluation of Urban Water System Metabolism Efficiency
}

\author{
Dr. Vladimir Nikolic ${ }^{1}$ and Prof. Darko Joksimovic \\ ${ }^{1}$ Ryerson University, Toronto, Ontario, Canada \\ nikolic.vladimir@ryerson.ca, darkoj@ryerson.ca
}

\begin{abstract}
The revitalization of Toronto's waterfront presents the largest urban redevelopment project currently underway in North America. With respect to planning the waterfront's urban water systems (UWS), a number of studies considered a range of criteria in search for sustainable alternatives. However, a comprehensive assessment of the integrated source-drinking-wastewater-stormwater systems over their life cycles has not been developed. According to the main postulates of the integrated approach, hybrid water systems can offer potentially more sustainable solutions than traditional centralized systems. This paper discusses the development process of a decision support tool designed to facilitate evaluation of alternatives based on UWS metabolism concept while addressing some typical challenges of hydroinformatics. This decision-making support tool analyses and compares the sustainability performance of alternative decentralized solutions against a baseline conventional approach on a neighbourhood level. The tool uses a set of criteria, adopted by the large group of stakeholders involved in the development process, that are not typically considered in the decision-making process, such as energy savings, greenhouse gas (GHG) emissions, climate change resiliency, chemical use, and nutrient recovery.
\end{abstract}

\section{Introduction}

Over the last several decades, a rapidly growing number of urban areas around the world have become challenged by a diverse set of issues associated with planning and management of municipal water resources and infrastructure. In addition to uncertainties caused by the more evident water shortages, drastically increased demands for water, stricter potable water standards, and destructive effects of climate variability, one particularly important trend can be detrimental to municipal water infrastructure if not addressed appropriately. Due to the significant growth of population on one side, and a lack of developable land on the other, cities like Toronto, Canada, are compelled to increase the 
rates of population density in urban cores. While environmentally justified, such intensification efforts raise numerous concerns as the scale of new developments and the range of different socio-economic activities within the existing systems challenge their ability to sustain and improve the quality of life for local residents. This leaves urban planners and designers seeking for tools and criteria that facilitate assessment of alternative solutions in an effort to plan and manage sustainable communities under constraining environmental conditions. The scope of this problem requires active collaboration of multiple decision makers, including environment specialists, designers, engineers, economists, government officials, and the local community, but also necessitates a clear sustainability framework for analyzing potential solutions and identifying the most preferred option considering both quantitative and qualitative criteria.

Developing decision support tools (DSTs) is one way how decision-makers can look for evidence of potential effectiveness or limitation of planning solutions. DSTs are typically used to form, test, and adjust theories related to many areas of city life planning. These theories are then tested against data observed and collected. Once the results adequately mimic reality, a DST utilizes modified inputs to examine potential outcomes under new conditions. Hydroinformatics plays a fundamental role in the development of such tools. As a field of applied informatics, hydroinformatics focuses on application of information and communications technologies in addressing the issues related to water management. However, in order to develop a tool that is useful, verified and reliable for analyzing outcomes of different urban water management practices, one must address a set of challenges distinctive for hydroinformatics. Firstly, the sources of complexity of water resources systems need to be represented systematically. Secondly, one needs to involve stakeholders in the tool development process to represent management practices and policies faithfully. Finally, one needs to develop an evaluation framework, which clearly analyzes, compares, and communicates alternatives by processing raw tool outputs.

This paper provides details how these challenges of hydroinformatics have been addressed during the development process for Integrated Water Resources Evaluation Tool (IWRET). IWRET presents a result of a collaboration between academia (Ryerson University, Toronto, Canada) and governmental institutions (Waterfront Toronto, Canada), in an endeavor of a participatory modelling and application of bottom-up modelling approach to address local water management issues. The main motivation for this collaboration and IWRET development lies in potential water management issues at Toronto's waterfront. The revitalization of Toronto's waterfront presents the largest urban redevelopment project currently underway in North America. A reclaimed, once industrial, land will bring thousands of new residents and jobs to Toronto's city core, further expanding the pressure on existing infrastructure. To steer the sustainable redevelopment of the waterfront, a comprehensive sustainability framework ensures that consistent principles are implemented into every aspect of decision-making. Nevertheless, while the proposed servicing options consider a range of criteria in determining preferred alternatives of a sustainable urban water system (UWS), the sustainability framework lacks a comprehensive assessment of the integrated source-drinking-wastewater-stormwater systems over their life-cycles [1]. Moreover, the existing sustainability framework, through existing master plans, considers only traditional, centralized solutions, and does not take into consideration the potential benefits of alternative, decentralized water technologies. Having that in mind, the key objective of IWRET is to expand the scope of existing framework and support decision-makers in analysis and comparison of the sustainability performance of alternative, decentralized approaches against a baseline conventional approach.

\section{Organization of the IWRET development process}

Similarly to the software development life cycle (a.k.a. software development process), the process of DST development is typically divided into a number of phases, where each phase comprises of a set 
of activities. Depending on the arrangement of phases, one can distinguish several types of software development processes: iterative process, agile software development, XP extreme programming, waterfall process, V-model, etc. What is important to distinguish is that each software development process is characterized by set of actions deployed in selected order, but it is typically iterative in nature, requiring constant reconsideration of completed tasks. On the other side, the scope of actions do not differ depending on the applied type, Table 1.

\begin{aligned} & \hline User Requirements Recognizes the basic needs of a potential user and defines goals of the tool. \\ & \hline System Design $\begin{array}{l}\text { Communication with area experts to facilitate better understanding of the } \\ \text { modeled phenomenon and their requirements. }\end{array} \\ &$\hline Specification $\begin{array}{l}\text { Describes technical details of the tool and layouts different scenarios of } \\ \text { interaction between the user and the tool. }\end{array} \\ & \begin{array}{r}\text { Abstract } \\ \text { Architecture }\end{array}$ Defines an abstract representation of the tool. \\ & \hline Implementation $\begin{array}{l}\text { Produces the actual code, along with the development of graphical user } \\ \text { interface (GUI). }\end{array} \\ &$\hline Model Testing Tests the produced code and corrects recognized errors. \\ & \hline Documentation $\begin{array}{l}\text { Describes the internal structure of the tool in order to provide a base for } \\ \text { future maintenance and enhancements. }\end{array} \\ &$\hline Deployment Distributes the tool to the user. \\ & \hline Training and For new and regular users of the tool. \\ & \hline Support $\begin{array}{l}\text { Addresses newly discovered problems and deals with emerging model } \\ \text { requirements. }\end{array} \\ &$\hline\end{aligned}

Table 1: List of actions in a software development process

\section{Scientific rationale, methodological basis and user requirements}

In the first phase of the DST development process, it is essential to identify the scientific rationale and define suitable methodological basis of the tool. Moreover, in this phase, the basic needs of a potential user and goals of the tool are recognized. In search for the scientific rationale for the tool, literature analysis showed that the process of planning, development and retrofitting of urban infrastructure is not only financially intensive, but also demanding in terms of water, energy, materials, or labor requirements. Based on similar observations, in 1965, an American scientist and sanitary engineer, Abel Wolman, envisioned the concept of urban metabolism as a holistic sustainability paradigm that takes into account all these aspects simultaneously, [2]. After years of dormancy, [3] have reintroduced the urban metabolism concept as the "sum total of the technical and socio-economic processes that occur in cities, resulting in growth, production of energy, and elimination of waste". With growing environmental concerns, the search for a more balanced urban metabolism has become a key element of sustainable performance in cities around the world.

From the perspective of urban water resources and infrastructure, according to [4], the importance of water-related fluxes in urban metabolism model is particularly emphasised since the urban water cycle has a rather substantial impact on flows of materials, energy, wastes and nutrients. Traditional urban water metabolism approach has largely focused only on centralized drinking, stormwater and wastewater systems, with the main intent of matching supply to demand. However, this centralized 
approach has become characterized by an increasing discrepancy between system entries and outflows causing considerable social, economic, and environmental impacts. In progressively resource limited cities, adopting new technologies and recreating urban morphology has become a paramount in achieving a sustainable water metabolism. The question remains what are the technologies that can be used to balance the urban water metabolic process, and what are their benefits, limitations and weaknesses?

A holistic approach can potentially offer a pathway to a long-term sustainability of urban water metabolic process. In order to deal with the mounting pressures over the valuable resource and complex systems of municipal water infrastructure, international water community has introduced a holistic approach named Integrated Urban Water Management (IUWM) [5]. Regarding planning and management of urban water infrastructure, IUWM approach encompasses supervision of all urban water cycle components, such as drinking water treatment, distribution, sewerage and storm drainage, and wastewater treatment. However, the reality for many cities around the world, including Toronto, is significantly different. One of the main legacies of the conventional approach is that urban areas rely predominantly on centralized water systems that typically consist of separate drinking water, stormwater and wastewater sub-systems. This fact embodies an enormous social and engineering issue for many urban developments, as further expansion of existing systems is neither economically nor environmentally viable. In view of that, IUWM suggests development of water systems that utilize alternative water sources, previously treated as nuisance, including rainwater, stormwater, greywater, or wastewater [6]. Within a built environment, there are four groups of decentralized solutions available to water utilities to achieve this: 1) Water-supply and water-demand management; 2) Low Impact Development (LID) and Green Infrastructure (GI); 3) Green buildings; and, 4) Greywater management and onsite reuse technologies.

While decentralized solutions can have different spatial scales of implementation (household, neighbourhood or city level), they generally operate alongside or in combination with centralized water systems and thus form the so-called hybrid water services systems. Due to its complexity, WIREs Water [7] argue that the design and management of hybrid water systems must take an integrated approach that accounts for the six disciplinary dimensions of an urban water system: Spatial and Temporal Variability; Energy and Greenhouse Gas (GHG) intensity; Downstream Impacts; Legal and Institutional; Social Aspects; and, finally, Economic Viability.

While utilization of decentralized technologies provides an opportunity for a number of benefits (such as cost reduction, resource efficiency, service security, system failure reduction, local economic strength, community wellbeing, and environmental protection), it typically involves the trade-offs between water use, energy use, land use and public concerns that must be taken into consideration. Perhaps the biggest challenge facing the implementation of hybrid systems is the lack of empirical information depicting system success and failure. Even though the approach of IUWM has gradually matured over time, one particularly important challenge has remained unresolved since the introduction; the development of tools for operational implementation of the concept and dynamic coupling of natural, engineering and socio-economic components of hybrid water systems. A literature review suggests that numerous simulation models have been developed in search for the optimal management strategies in different contexts, such as MUSIC [8], PURSS [9], Urban Volume and Quality [10], UrbanCycle [11], Urban Developer [12], MikeURBAN [13], and SUSTAIN [14], and UrbanBEATS [15].

A substantial number of these models looks at these components in isolation, but the sustainable implementation of hybrid water systems requires a more comprehensive and integrative tool that takes into account all these aspects simultaneously. In order to challenge this, literature suggests there have been a number of mathematical urban water metabolism models developed for specific case studies, such as urban metabolism models developed for Canadian cities [16]. In contrast, the two most prominent generic modelling frameworks that can be adapted and applied to different urban settings are Urban Water Optioneering Tool (UWOT) [17] and WaterMET ${ }^{2}[18]$. 
Having that in mind, the key IWRET user requirement is that it represents a tool that can assist planners, utility managers, engineers, and other decision-makers determine the optimal trade-offs in order to support sustainability at the community level. IWRET analyses and compares the sustainability performance of alternative approaches to integrated urban water management strategies and technologies against a baseline conventional approach. Therefore, IWRET is mass-balance model at core, built on the concept of urban water metabolism that takes into consideration water, energy and nutrient flow, Figure 1. IWRET focuses on the intermediate level (new development, community or neighbourhood) and investigates the impacts of decentralized urban water management technologies on a number of indicators, such as energy savings, greenhouse gas emissions, climate change resiliency, chemical use, and nutrient recovery.
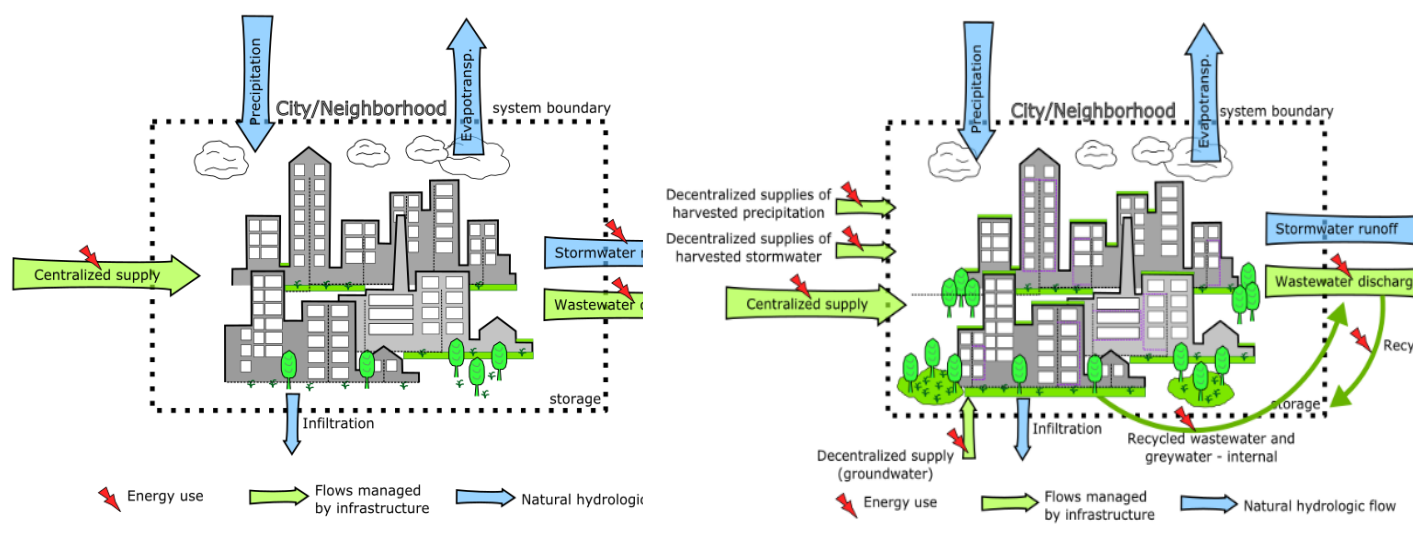

Figure 1: Traditional (Centralized) and Alternative (Decentralized) Urban Water Metabolism Process

From an operational perspective, IWRET should address one of the major challenges in hydroinfromatics; a comprehensive representation of complex, interconnected water resources systems. This particular objective is achieved by:

- Integrating all elements of the urban water cycle (drinking water, stormwater, wastewater, and recycle/reuse);

- Including all four groups of decentralized (distributed) solutions to allow flexible representation of hybrid water systems;

- Considering all six disciplinary dimensions (Spatial and Temporal Variability; Energy and GHG intensity; Downstream Impacts; Legal and Institutional; Social Aspects; and, finally, Economic Viability);

- Building the model on the open-source technology, publicly available for use, modification and distribution;

- Incorporating low data requirements typically used for master planning process; and,

- Integrating of sustainability performance indicators recognized by the potential users.

\section{Direct stakeholder involvement in the design process}

Second challenge of hydroinformatics in terms of adequate representation of water management practices is active involvement of stakeholders and potential users in the model development process. The reason for this is that, on occasion, a model developer is not sufficiently educated on specific domain where the tool is applied. Therefore, in the second phase of a tool development, it is crucial to 
communicate with area experts and facilitate better understanding of their requirements. In an effort to achieve the structural accuracy and validity of the tool, more than 50 stakeholders (area experts from governmental institutions, technology developers, providers and distributors, developers and builders, consultants, and non-governmental organizations) were engaged in an IWRET development workshop. Workshop participants were given an opportunity to give direct input on the tool development by providing the direct feedback on:

- Anticipated decentralized technologies to be included in the tool;

- Harmonization of quantitative and qualitative sustainability indicators for the evaluation of alternative solutions; and, finally,

- Preference on the features of tool's graphical user interface (GUI).

Firstly, workshop participants were introduced to a set of decentralized urban water technologies divided into three groups (water supply, wastewater, and stormwater), including their integration through recycling/reuse. Participants then prioritized distributed technologies on a scale 1 - not important, to 5 - very important. The group of stakeholders recognized water saving devices as the most important technology to be included in IWRET, followed by smart water meters and integration of smart water and energy metering, Figure 2. On the other side, downspout disconnections and stormwater reuse systems were considered the most important distributed stormwater technologies, Figure 3. Regarding wastewater distributed systems, packaged wastewater treatment plants and constructed wetlands were valued as the most significant technologies, Figure 4. Finally, greywater systems and rainwater harvesting technologies were valued as the most important technologies to be included in IWRET for the recycling/reuse side of the urban water cycle, Figure 5.

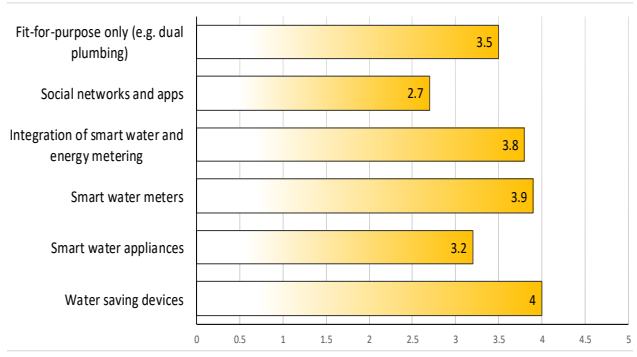

Figure 2: Distributed water supply technologies

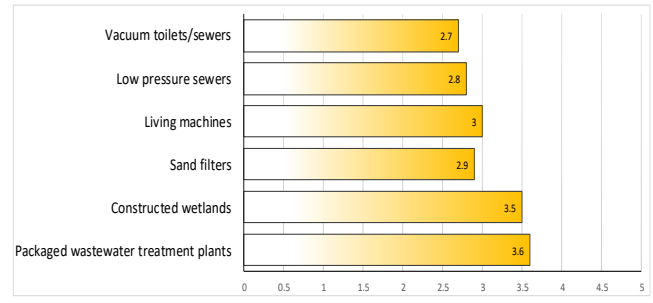

Figure 4: Distributed wastewater technologies

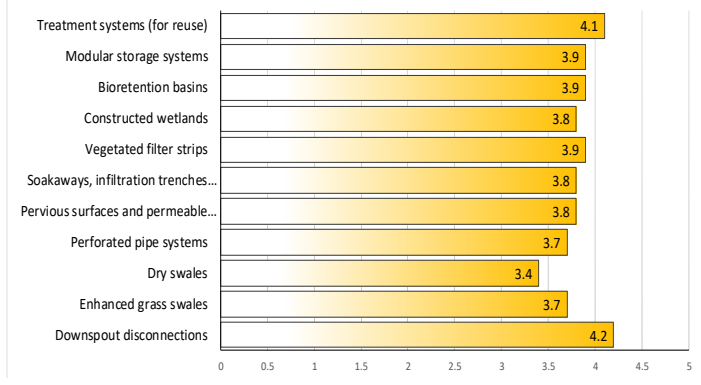

Figure 3: Distributed stormwater technologies

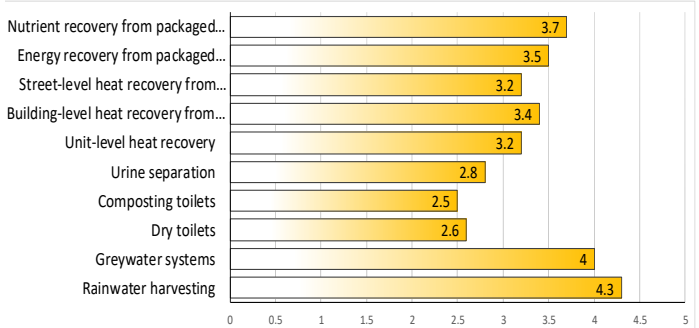

Figure 5: Reuse/recycle technologies

One of the major challenges in water systems engineering practice is the development of tools to quantify and enhance urban infrastructure sustainability planning, design and management. The integration of sustainability assessment into decision-making processes is therefore becoming an essential task for water service providers. Typically, quantifying sustainability is an ambiguous process. It requires considerable attention to respond to the particular characteristics of the problem at hand. While various categories of sustainability criteria have been suggested to evaluate alternative solutions, literature suggests that they can be categorized into a four primary groups including economic, environmental, engineering and socio-cultural. Since they are strongly context driven, workshop 
participants were presented with a preliminary list of sustainability indicators based on similar tools developed around the world.

Participants identified life-cycle costs and operational costs as the most dominant factors to sustainable water infrastructure planning. This result suggests that IWRET should carefully consider financial aspects of operation and maintenance costs. When it comes to the analysis of the return on investment, several factors must be looked at, primarily the payback period instead of the quality of the investment itself. The main recommendation is to focus on operational rather than capital cost. Finally, the participants recommended that IWRET should be able to optimize operational expenditure, capital expenditures, and lifecycle costs, and that it should add incentives from the municipality (e.g. capital cost reduction, economic incentives, or quicker approval) if the project is to proceed to more detailed stages of planning and design. Participants further identified performance and reliability of assets amongst the most important indicators. The participants expressed the opinion that IWRET should include dynamic analysis of device performances (e.g. operations of pumps). Amongst the most important environmental indicators were rainwater runoff, both quality and quantity, in addition to savings in wastewater generation and indicators related to energy use and energy reductions. Finally, among social indicators the potential risk to human health is the indicator of highest importance.

The final objective of the workshop was to receive input from the stakeholders to inform the development of IWRET graphical user interface (GUI). Based on a poll taken during the workshop, $55 \%$ of participants preferred a user-friendly interface built within a familiar platform (such as Excel) that would incorporate user feedback and it should include not more than limited representation of spatial variability of UWS.

\section{Abstract architecture of the tool}

Once the methodological basis is laid out (metabolic performance of urban water systems, both centralized and hybrid) and the main requirements of the tool are adopted (technologies, sustainability criteria, and preferred GUI), the next phase of the tool development process defines the abstract architecture of the model and describes interaction between the user and the model. Dictated by the very restricted representation of spatial variation of UWS requested by the stakeholders, system dynamics (SD) is considered the most suitable modelling method for creating the numerical core of the model. SD simulation is a modelling method commonly used in IUWM for its capacity to describe complex relations between different components of water systems [19]. It utilizes the principles of the feedback control theory to form simulation models, while a feedback loop presents the core building block. From the water resources management perspective, it offers a number of advantages [20]. Firstly, it allows incorporation of engineering, social, economic and environmental elements into the equation. Secondly, it provides a direct link between a structure and behaviour of a system, allowing further examination of the change in system structure and its effects on system behaviour. Finally, it promotes an active involvement of all interested parties and stakeholders in the modelling process.

Stocks and flows represent the core of all SD models, Figure 6. While flows represent the rates of change in time (such as $\mathrm{m}^{3} / \mathrm{day}$, or $\mathrm{kg} / \mathrm{m}^{3}$ ), the stocks can be described as accumulations or quantities (i.e. $\mathrm{m}^{3}, \mathrm{~kg}$, etc.). In a mathematical sense, stocks are simply integral equations [21]. These accumulations drive the model toward equilibrium as they stabilize the whole system. 


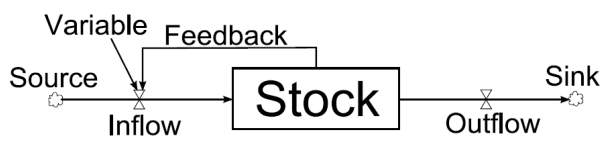

Figure 6: Main elements of System Dynamics (SD) simulation model

A third challenging issue with the DST development process is that obtained simulation results require post-processing for analysis, communication and clear comparison of alternatives. In order to provide a user-friendly GUI, IWRET is being developed as a standalone piece of software written in Python programming language. PySD package is used to simulate SD models in Python [22]. PySD translates SD model files into Python modules, and provides methods to simulate and analyse converted models. For GUI development, PyQt is used [23]. PyQt is a Python binding of the cross-platform GUI toolkit Qt, and is free for non-commercial use.

In many aspects, the unsustainable nature of modern cities is a consequence of poor planning at the neighbourhood level. The idea is that the development of sustainable neighbourhoods helps achieve a sustainable urban form at the macro level. This is the justification for IWRET to support strategic planning of urban water systems on the neighborhood level. In terms of temporal resolution, IWRET uses a daily time step to simulate the performance of urban water systems for the specified period.

In order to conduct a comparative analysis of metabolic performance for the two different approaches, on the main program window, the user describes properties of both a traditional (centralized) option and a hybrid option. Each option has a set of forms that refer to all components of an urban water system. Input data forms are used for defining an operational IWRET model. The main components of an urban water system represented include:

- Water Treatment Form - defines metabolic features of water treatment plant;

- Conveyance, Trunk and Distribution Mains Form - defines metabolic features of trunk and distribution mains;

- Hydrology and LID Form - defines hydrologic component urban water system;

- Sewer System Form - defines metabolic features of sewer system;

- Stormwater System Form - defines metabolic features of stormwater system;

- Stormwater Treatment Form - defines metabolic features of stormwater treatment;

- Wastewater Treatment Plant Form - defines metabolic features of wastewater treatment plant;

- Pumping Stations Form - defines metabolic features of water, wastewater and stormwater pumping system;

- Reuse/Recycle Form - defines metabolic features of reuse/recycle systems

\section{Conclusions}

This paper details on a collaboration between academia, government, and a group of stakeholders, in a participatory, bottom-up modelling approach. Integrated Water Resources Evaluation Tool (IWRET) is being developed during this collaboration. IWRET is envisioned as a tool that assists planners, utility managers, engineers, and other decision-makers to determine the benefits and tradeoffs of hybrid water systems in order to support sustainability master planning at the community level. IWRET analyses and compares the performance of alternative, hybrid approaches, strategies and technologies against a baseline conventional approach. At its core, IWRET is mass-balance-based model, built on the concept of urban water metabolism, which takes into consideration water, energy and nutrient flow. IWRET focuses on the intermediate level (neighbourhood) and investigates the 
impacts of decentralized technologies on a number of indicators, such as energy savings, greenhouse gas emissions, climate change resiliency, chemical use, and nutrient recovery.

Moreover, the process of IWRET development tries to address three typical challenges of hydroinformatics. The first one is to represent the sources of complexity of water resources systems. The second is to actively involve stakeholders in the model development process to represent management practices and policies authentically. Finally, the third is to develop an evaluation framework, which clearly analyzes, compares, and communicates alternatives by processing raw model results.

IWRET systematically represents the sources of complexity of water resources systems by taking the metabolism approach, and:

- Considering all components of the urban water system by incorporating the feedback based system structure and their non-linear interactions;

- Modeling factors that change with time (demand, demographics, land use, system configuration and capacity, etc.);

- Involving representation of water conservation technologies (green infrastructure, green buildings and recycling);

- Establishing the feedback links with other environmental cycles (e.g. energy, greenhouse gases and nutrients);

- Providing support for direct stakeholder participation and involvement.

The feedback from the interested stakeholders is used to inform the development of the tool architecture, including anticipated technologies to be represented in the tool, selection of quantitative and qualitative sustainability indicators for the evaluation of alternative solutions, and, lastly, the general preference of the preferred tool's graphical user interface (GUI). Current effort is on the actual tool development following one of the driving project requirements: to develop a tool that is transferable, non-proprietary and applicable to other neighborhoods, jurisdictions and municipal settings. Once it is developed, IWRET will be applied to a newly created community at Villiers Island, Toronto to optimize the utilization of water, energy and nutrients for the proposed development. IWRET will be used to define a number of alternative strategies that include decentralized options for water, wastewater, drainage, and recycling. The strategies will vary from the traditionally centralized baseline to fully decentralized systems. Baseline strategy assumes application of current practices with centralized water service systems that do not include recycling or reuse. This scenario is driven by currently valid regulations on municipal and provincial level. On the other hand, fully decentralized strategy incorporates solutions under environmental constraints, including severe limitations in the wastewater system capacity or severe limitations in the water supply system capacity.

\section{References}

[1] Waterfront Toronto http://www.waterfrontoronto.ca/Sustainability (Accessed March, 2018)

[2] A.Wolman, The metabolism of cities. Scientific American 213 (3), (1965) 179-190.

[3] C. Kennedy, J. Cuddihy, J. Engel-Yan, the Changing Metabolism of Cities. Journal of Industrial Ecology (2007) 11(2) 43-59.

[4] P. Baccini, A city's metabolism: towards the sustainable development of urban systems. Journal of Urban Technology (1997), 4 (2), 27-39.

[5] J. S. Thomas, B. Durham, Integrated water resource management: looking at the whole picture, Desalination 156 (2003) 21-28.

[6] V. Novotny, J. Ahern, P. Brown, Water Centric Sustainable Communities, John Wiley and Sons, Inc., Hoboken, New Jersey, 2010.

[7] WIREs Water Report (2015), 2:623-634. doi: 10.1002/wat2.1099 
[8] eWater, http://www.ewater.org.au/products/music/ (Accessed March, 2018)

[9] P.J. Coombes, Integrated Water Cycle Modelling Using PURRS (Probabilistic Urban Rainwater and wastewater Reuse Simulator), Urban Water Cycle Solutions, (2006)

[10] V.G. Mitchell, C. Diaper, S.R. Gray, M. Rahili, UVQ: Modelling the Movement of Water and Contaminants through the Total Urban Water Cycle. In: 28 International Hydrology and Water Resources Symposium, Wollongong, NSW, (2007)

[11] M.J. Hardy, G. Kuczera, P.J. Coombes, Integrated urban water cycle management: the UrbanCycle model. Water Science \& Technology, 52 (2005) (9): 1-9. 2018)

[12] eWater http://ewater.org.au/products/music/related-tools/urban-developer/ (Accessed March,

[13] MIKE URBAN https://www.mikepoweredbydhi.com/products/mike-urban (Accessed March, 2018)

[14] U.S. EPA. SUSTAIN, A Framework for Placement of Best Management Practices in Urban Watersheds to Protect Water Quality. (Publication No. EPA/600/R-09/095, September 2009), (2009)

[15] P. M. Bach, UrbanBEATS - A virtual urban water system tool for exploring strategic planning scenarios. Doctor of Philosophy, Monash University. Melbourne, Australia, 2014

[16] H. R. Sahely, S. Dudding, C. A. Kennedy, Estimating the urban metabolism of Canadian cities: Greater Toronto Area case study, Can. J. Civ. Eng., (2003) 30: 468-483

[17] C. K. Makropoulos, K. Natsis, S. Liu, K. Mittas, D. Butler, Decision support for sustainable option selection in integrated urban water management, Environ. Modell. Software (2008), 23(12), 1448-1460,

[18] K. Behzadian, Z. Kapelan, G. Venkatesh, H. Brattebø, S. Sægrov, WaterMet²: a tool for integrated analysis of sustainability-based performance of urban water systems. Drinking Water Engineering and Science (2014), 7(1), 63-72

[19] I. Winz, G. Brierley, S. Trowsdale, The Use of System Dynamics Simulation in Water Resources Management. Water Resources Management (2008), 23(7), 1301-1323. doi:10.1007/s11269-008-9328-7

[20] S.P. Simonovic, Managing water resources: methods and tools for a systems approach, UNESCO, Paris and Earthscan James \& James, London, (2009) ISBN 978-1-84407-554-6

[21] D. Meadows, Thinking in Systems: A Primer, Chelsea Green Publishing (2008) ISBN-13: 9781603580557

[22] PySD http://pysd.readthedocs.io/en/master/ (Accessed March, 2018)

[23] PyQt https://riverbankcomputing.com/software/pyqt/intro (Accessed March, 2018) 